\title{
A Generalized Ginzburg-Landau Approach to Second Harmonic Generation
}

\author{
Debanand Sat \\ Institut für Physik, Universität Dortmund, 44221 Dortmund, Germany \\ R. Valentí and C. Gros \\ Fachbereich Physik, University of the Saarland, 66041 Saarbrücken, Germany
}

\begin{abstract}
We develop a generalized Ginzburg-Landau theory for second harmonic generation (SHG) in magnets by expanding the free energy in terms of the order parameter in the magnetic phase and the susceptibility tensor in the corresponding high-temperature phase. The non-zero components of the SHG susceptibility in the ordered phase are derived from the symmetries of the susceptibility tensor in the high-temperature phase and the symmetry of the order parameter. In this derivation, the dependence of the SHG susceptibility on the order parameter follows naturally, and therefore its nonreciprocal optical properties. We examine this phenomenology for the magnetoelectric compound $\mathrm{Cr}_{2} \mathrm{O}_{3}$ as well as for the ferroelectromagnet $\mathrm{YMnO}_{3}$.
\end{abstract}

PACS Numbers: 42.65.-k: Nonlinear Optics, 75.50.Ee: Antiferromagnetics

Second Harmonic Generation (SHG) is a very useful technique to study the nonlinear optical properties [1] in magnetic materials. The recent observation [2.3] of nonreciprocal optical effects (i.e., not invariant under time reversal operation) in the magnetoelectric material $\mathrm{Cr}_{2} \mathrm{O}_{3}$ below the Néel temperature $T_{N}$ has been of great importance in the study of antiferromagnetic (AFM) ordering by light. With the help of SHG, photographs of the antiferromagnetic domains [3, 4 , in $\mathrm{Cr}_{2} \mathrm{O}_{3}$ were obtained what confirms that these experiments can distinguish between the two magnetic states that are related to each other by the time reversal operation, and therefore its nonreciprocity. These observations can be explained by an interference effect between a time-symmetric magnetic dipole contribution and a time-antisymmetric electric dipole contribution [3]. Soon after these experiments were done, a microscopic theory was proposed 5 which could explain quantitatively the non-reciprocal effects observed in $\mathrm{Cr}_{2} \mathrm{O}_{3}$. It was shown that the electric dipole contributions were linearly proportional to the antiferromagnetic order parameter giving rise to the timeantisymmetric character to the electric dipole tensor. The study of this dependence is of crucial importance in order to understand the non-reciprocal character of the SHG tensors. The derivation of a micoscopic theory for a specific effect, in our case SHG, can be rather complicated depending on the properties of the material under study [5.6]. Concrete information of the SHG process in materials where a transition takes place, i.e. paramagnetantiferromagnet or paraelectric- ferroelectric, can be obtained at a simpler level, i.e. by considering only the symmetry arguments. In particular, we are interested in investigating the dependence of the SHG susceptibilities in the ordered phase on the order parameter.

A powerful phenomenological theory suitable to describe phase transitions is the Ginzburg-Landau theory. The Ginzburg-Landau approach is based on the existence of an order parameter in the ordered phase and on symmetry considerations [7]. Pershan in 1963 [8] showed that the tensors for nonlinear electro- and magneto-optic effects could be derived from a phenomenological timeaveraged free energy. It is our purpose in this paper to extend the formulation of Pershan by including the order parameter explicitly in the expansion of the free energy for SHG. We shall show that the non-zero components of the SHG susceptibility tensor in the ordered phase are naturally obtained from the symmetry of the susceptibility tensor in the high-temperature phase and the symmetry of the order parameter. Once the dependence of the SHG susceptibility tensor on the order parameter is known, the non-reciprocal optical properties below the transition temperature follow. We explicitly verify this formulation in the magnetoelectric compound $\mathrm{Cr}_{2} \mathrm{O}_{3}$ as well as the hexagonal ferroelectric-antiferromagnetic material $\mathrm{YMnO}_{3}$.

A convenient starting point in order to describe the optical nonlinearities is given by a time-averaged free energy $F$ as proposed by Pershan [8]. A dipole expansion of the induced current $\mathbf{J}=\partial \mathbf{P} / \partial t+c \nabla \times \mathbf{M}-\partial(\nabla \cdot \mathbf{Q}) / \partial t+\ldots$, where $\mathbf{P}, \mathbf{M}$ and $\mathbf{Q}$ are respectively the electric dipole polarization, the magnetization and the electric quadrupole polarization, leads to [8]

$$
F=-2 \operatorname{Re} \sum_{\nu=1}^{n}\left[\mathbf{E}^{*}\left(\omega_{\nu}\right) \cdot \mathbf{P}\left(\omega_{\nu}\right)+\mathbf{H}^{*}\left(\omega_{\nu}\right) \cdot \mathbf{M}\left(\omega_{\nu}\right)\right]
$$

where we have discarded the electric quadrupole and higher-order terms and a constant contribution. Here $\mathbf{E}\left(\omega_{\nu}\right), \mathbf{P}\left(\omega_{\nu}\right), \mathbf{H}\left(\omega_{\nu}\right)$ and $\mathbf{M}\left(\omega_{\nu}\right)$ are respectively the Fourier components of the electric field, electric dipole polarization, magnetic field and the magnetization and $\nu$ denotes the number of partial waves. The usual relations

$$
\begin{aligned}
\mathbf{P}\left(\omega_{\nu}\right) & =-\partial F / \partial \mathbf{E}^{*}\left(\omega_{\nu}\right) \\
\mathbf{M}\left(\omega_{\nu}\right) & =-\partial F / \partial \mathbf{H}^{*}\left(\omega_{\nu}\right)
\end{aligned}
$$

are fullfilled. The electric dipole polarization and the magnetization are, in general, nonlinear functions of $\mathbf{E}$ 
(and $\mathbf{H})$ which may be expanded into a power series in $\mathbf{E}$ (and $\mathbf{H}$ ). The second order term for the electric dipole polarization is

$$
P_{i}(2 \omega)=\chi_{i j k}^{(E D)}(2 \omega, \omega, \omega) E_{j}(\omega) E_{k}(\omega)
$$

which corresponds to the SHG electric dipole contribution (ED). The corresponding free energy is then (see Eq. (11)):

$$
\begin{aligned}
F^{(E D)} & =-\left[\chi_{i j k}^{(E D)}(2 \omega, \omega, \omega) E_{i}^{*}(2 \omega) E_{j}(\omega) E_{k}(\omega)\right. \\
& \left.+\chi_{i j k}^{*}{ }^{(E D)}(2 \omega, \omega, \omega) E_{i}(2 \omega) E_{j}^{*}(\omega) E_{k}^{*}(\omega)\right]
\end{aligned}
$$

Summation over repeated indices is implicit in the above formulas.

As a second example, we consider the magnetic dipole contribution (MD) to SHG,

$$
M_{i}(2 \omega)=\chi_{i j k}^{(M D)}(2 \omega, \omega, \omega) E_{j}(\omega) E_{k}(\omega),
$$

leading to

$$
\begin{aligned}
F^{(M D)} & =-\left[\chi_{i j k}^{(M D)}(2 \omega, \omega, \omega) H_{i}^{*}(2 \omega) E_{j}(\omega) E_{k}(\omega)\right. \\
& \left.+\chi_{i j k}^{*}{ }^{(M D)}(2 \omega, \omega, \omega) H_{i}(2 \omega) E_{j}^{*}(\omega) E_{k}^{*}(\omega)\right] .
\end{aligned}
$$

$F^{(M D)}$ is the free energy corresponding to the MD contribution to SHG. Both the ED and the MD contribute to the source term S, i.e., as $\mu_{0}\left[\left(\partial^{2} P / \partial t^{2}\right)+\nabla \times(\partial M / \partial t)\right]$ in the wave equation for the electric field, derived from the Maxwell's equations in classical electrodynamics [3]. The measured output intensity in a nonlinear optical experiment is $I \propto|S|^{2}$ and once the SHG susceptibility in the ordered phase is known, possible non-reciprocal properties of the system may be derived.

In the context of the antiferromagnetic $\mathrm{Cr}_{2} \mathrm{O}_{3}$ it has been observed [3] that $\chi_{i j k}^{(E D)}$ exists only in the ordered phase $\left(T<T_{N}\right)$ and it has been shown [5.60 that it is linearly proportional to the antiferromagnetic order parameter, as it should be if we had defined a Ginzburg-Landau free energy for the ordered phase. In the framework of the classical Ginzburg-Landau approach to phase transitions the allowed terms contributing to the free energy follow from the symmetry of the order-parameter and from the symmetry of the lattice [7,9]. It is therefore of interest to ask oneself whether one can combine the standard Ginzburg-Landau approach for the magnetic properties of e.g. $\mathrm{Cr}_{2} \mathrm{O}_{3}$ with the expressions Eq. (3) and Eq. (5) in order to obtain a more general formulation for the nonlinear magneto-optical properties of a given compound.

In order to generalize the Ginzburg-Landau formulation to study the nonlinear optical properties in antiferromagnets we first note that the (generalized) GinzburgLandau functional has to obey the symmetry of the hightemperature phase, as the (spontaneous) breaking of this symmetry is inherent in the solution which is a minimum of the Ginzburg-Landau functional. As an illustration, we consider a hypothetical magnetic dipole contribution to SHG which is only present in the ordered phase. Let us assume the following symmetries for the order parameter. i) it is a c-tensor, i.e. antisymmetric under the time reversal operation and ii) it is a (real) axial tensor of first rank ( a pseudovector), i.e. $O_{l}$. In this case

$$
\begin{aligned}
F & =-\left[\chi_{i j k l}\left(T>T_{N}\right) H_{i}^{*} E_{j} E_{k}\right. \\
& \left.+\chi_{i j k l}^{*}\left(T>T_{N}\right) H_{i} E_{j}^{*} E_{k}^{*}\right] O_{l}
\end{aligned}
$$

would be a valid expression for the combined free-energy, where $\chi_{i j k l}\left(T>T_{N}\right)$ is the susceptibility tensor in the paramagnetic phase. The expression in the parenthesis indicates that the susceptibility is above the transition temperature $T_{N}$. It should be noted here that the free energy is a i-scalar, i.e., a scalar invariant under the time reversal operation. This implies that the susceptibility tensor in the paramagnetic phase has to be a polar itensor of fourth rank. Now, comparing Eq. (6) with Eq. (5), one would obtain the following relation between the SHG susceptibility and the order parameter:

$$
\chi_{i j k}^{S H G}\left(T<T_{N}\right)=\chi_{i j k l}\left(T>T_{N}\right) O_{l}
$$

It is clear from the above expression that from the knowledge of the order parameter and the symmetries of the susceptibility tensor $[10$ in the paramagnetic phase, it is possible to obtain all the non-zero components of the SHG susceptibility in the ordered phase. Moreover, the SHG susceptibility tensor below the transition temperature becomes directly proportional to the order parameter, which will ultimately manifest in the non-reciprocal optical effects in the system. In what follows, we examine this in detail for the cases of the magnetoelectric compound $\mathrm{Cr}_{2} \mathrm{O}_{3}$ and of the ferroelectric-antiferromagnetic material $\mathrm{YMnO}_{3}$.

$\mathrm{Cr}_{2} \mathrm{O}_{3}$, in its paramagnetic phase (above $T_{N} \approx 307 \mathrm{~K}$ ), crystallizes in the centrosymmetric point group $\overline{3} \mathrm{~m}$. The unit cell contains four $\mathrm{Cr}^{3+}$ ions, which occupy equivalent c-positions along the optical axis. This structure has a centre of inversion and parity considerations allow only axial i-tensors of odd rank and polar i-tensors of even rank. Thus, above $T_{N}$, SHG electric dipole effects are forbidden but magnetic dipole effects are allowed. Below $T_{N}$, the four spins in the unit cell order along the optical axis in a non-centrosymmetric AFM structure (the spin order being, up, down, up, down) which leads to two types of domains transformed into each other by time reversal symmetry. Both, space and time reversal symmetry operations are separately broken by the spin ordering but the combination of both the symmetries remains a symmetry of the crystal. The magnetic point group of $\mathrm{Cr}_{2} \mathrm{O}_{3}$ being $\underline{3} m$ allows new tensors, i.e., polar c-tensors of odd rank and axial c-tensors of even rank. Thus, electric dipole effects due to polar c-tensors of odd rank are allowed below $T_{N}$.

In order to construct the nonlinear free energy for $\mathrm{SHG}$, one needs to know the order parameter in $\mathrm{Cr}_{2} \mathrm{O}_{3}$. 
This can be achieved following Dzialoshinskii's 11] derivation for the Landau theory of second order phase transitions [9]. We assign a mean spin $\mathbf{S}_{\beta}(\beta=1,2,3,4)$ to each of the four $\mathrm{Cr}^{3+}$ ions. Out of the $\mathbf{S}_{\beta}$ one can form the following linear combinations $\mathbf{m}, \mathbf{l}_{\mathbf{1}}, \mathbf{l}_{\mathbf{2}}$ and $\mathbf{l}_{\mathbf{3}}$ :

$$
\begin{aligned}
\mathbf{m} & =\mathbf{S}_{1}+\mathbf{S}_{2}+\mathbf{S}_{3}+\mathbf{S}_{4} \\
\mathbf{l}_{1} & =\mathbf{S}_{1}-\mathbf{S}_{2}-\mathbf{S}_{3}+\mathbf{S}_{4} \\
\mathbf{l}_{2} & =\mathbf{S}_{1}-\mathbf{S}_{2}+\mathbf{S}_{3}-\mathbf{S}_{4} \\
\mathbf{l}_{3} & =\mathbf{S}_{1}+\mathbf{S}_{2}-\mathbf{S}_{3}-\mathbf{S}_{4}
\end{aligned}
$$

The components of the vectors $\mathbf{m}$ and $\mathbf{l}_{\alpha}(\alpha=1,2,3)$ can be classified according to the irreducible representations of the paramagnetic group $\overline{3} m$ as given in Table 1 [9,12]. The components $m_{z}, l_{1 z}, l_{2 z}, l_{3 z}$ transform according to the one-dimensional representations $A_{2 g}, A_{1 g}$, $A_{1 u}, A_{2 u}$ of the point group $\overline{3} m$. The $x$ and $y$ components of the vectors $\mathbf{m}$ and $\mathbf{l}_{\mathbf{1}}$ transform according to the two-dimensional representation $E_{g}$; and the $x$ and $y$ components of the vectors $\mathbf{l}_{\mathbf{2}}$ and $\mathbf{l}_{\mathbf{3}}$ transform according to $E_{u}$. We make now the following key observation:

The order parameter of a magnet is given by that irreducible representation of the paramagnetic point group which is invariant under the symmetries of the magnetic group.

This statement can be considered as a generalization of von Neumann's principle (see [10]) and follows from the observation that the thermodynamic expectation value of any combination of the constituent spins $\mathbf{S}_{\beta}$ which is not invariant under the magnetic group, i.e. $\underline{\overline{3} m}$ for the case of $\mathrm{Cr}_{2} \mathrm{O}_{3}$, can be shown to vanish identically. For $\mathrm{Cr}_{2} \mathrm{O}_{3}$ the irreducible representation which is invariant under $\underline{\overline{3} m}$ is $A_{1 u}$, i.e. $l_{2 z}$.

Thus, the order parameter for $\mathrm{Cr}_{2} \mathrm{O}_{3}$, which is the staggered magnetization constructed from $l_{2 z}$ is a c-axial scalar. The description given by $l_{2 z}$ corresponds to a collinear ordering (up,down,up,down) which is the spin ordering in $\mathrm{Cr}_{2} \mathrm{O}_{3}$. Now, we can write down the free energy due to the electric dipole contribution as,

$$
\begin{aligned}
& F=-\left[\chi_{i j k}\left(T>T_{N}\right) E_{i}^{*} E_{j} E_{k}\right. \\
& \left.\quad+\chi_{i j k}^{*}\left(T>T_{N}\right) E_{i} E_{j}^{*} E_{k}^{*}\right] l_{2 z}
\end{aligned}
$$

and therefore the SHG susceptibility tensor can be obtained as,

$$
\chi_{i j k}^{S H G}\left(T<T_{N}\right)=\chi_{i j k}\left(T>T_{N}\right) l_{2 z} .
$$

i.e. a c-polar tensor of third rank is obtained from the tensorial product of an i-axial tensor of third rank with a c-axial scalar (or pseudoscalar). Since the susceptibility tensor $\chi_{i j k}$ above $T_{N}$ is an i-axial tensor of third rank, we know all the non-zero components from the symmetry analysis [10]. Thus, using Eq. (10), one obtains all the non-zero components of the SHG $\chi$, which are, $\chi_{y y y}=-\chi_{y x x}=-\chi_{x y x}=-\chi_{x x y}$. Furthermore, the symmetry of $\chi\left(T>T_{N}\right)$ in the paramagnetic phase dictates that $\chi^{S H G}\left(T<T_{N}\right)$ is linearly dependent on the order parameter, which is the reason why one observes the AFM domains through SHG in $\mathrm{Cr}_{2} \mathrm{O}_{3}$.

The second example we want to illustrate is $\mathrm{YMnO}_{3}$. $\mathrm{YMnO}_{3}$ is a ferroelectromagnetic material whose crystal structure above the Curie temperature $T_{c} \approx 913 \mathrm{~K}$ is presumably centrosymmetric and described by the point group $6 / \mathrm{mmm}$. The elementary unit cell contains six $\mathrm{Mn}^{3+}$ ions. Below $T_{c}, \mathrm{YMnO}_{3}$ orders ferroelectrically and the charge ordering breaks inversion symmetry. The $\mathrm{YMnO}_{3}$ structure is then described by the non-centrosymmetric point group $6 \mathrm{~mm}$. The vector $\mathbf{P}^{(S P)}=\left(0,0, P_{z}{ }^{(S P)}\right)$ of spontanous polarization is directed along the six-fold z-axis. The magnetic properties of $\mathrm{YMnO}_{3}$ arise from the manganese ions $\mathrm{Mn}^{3+}$ in the high spin state $S=2$. Below the Néel temperature, $T_{N} \approx 74 K$, the spins of the six $\mathrm{Mn}^{3+}$ ions in the unit cell are ordered antiferromagnetically in a triangular structure perpendicular to the polar axis. The crystallographic, magnetic and electric properties of the hexagonal $\mathrm{YMnO}_{3}$ and the other rare-earth manganites were studied in the sixties and the related data are available in 13. New data concerning dielectric, magnetic, infrared and Raman studies have been also reported recently [14,15].

In the electric dipole approximation, SHG is allowed in $\mathrm{YMnO}_{3}$ below $T_{c}$ due to the inversion symmetry breaking by the ferroelectric ordering of charges [16]. Thus, from the symmetry of the susceptibility tensor in the paraelectric phase and that of the order parameter which is the spontaneous polarization $P_{z}^{(S P)}$ along the six-fold axis, one should be able to write down a free energy like Eq. (9). $6 / \mathrm{mmm}$ is centrosymmetric, which implies that in the paraelectric phase only polar tensors of even rank and axial tensors of odd rank are allowed. $P_{z}^{(S P)}$ is an i-polar first rank tensor (vector) directed along $z$. Therefore, in order to obtain an i-polar third rank tensor in the ferroelectric phase which should describe the SHG electric dipole contribution, only contractions of the $6 / \mathrm{mmm}$ symmetry polar tensors with odd powers of the order parameter are allowed. In the lowest order, the free energy can be written as,

$$
\begin{gathered}
F=-\left[\chi_{i j k z}\left(T>T_{c}\right) E_{i}^{*} E_{j} E_{k}\right. \\
\left.+\chi_{i j k z}^{*}\left(T>T_{c}\right) E_{i} E_{j}^{*} E_{k}^{*}\right] P_{z}^{(S P)}
\end{gathered}
$$

such that the SHG susceptibility tensor in the ferroelectric phase is obtained as,

$$
\chi_{i j k}^{S H G}\left(T_{c}>T>T_{N}\right)=\chi_{i j k z}\left(T>T_{c}\right) P_{z}^{(S P)} .
$$

Here the susceptibility tensor $\chi_{i j k l}$ above the Curie temperature $T_{c}$, i.e. in the paraelectric phase, is an i-polar tensor of fourth rank. Now, from the symmetry analysis [10] of this tensor, one gets the non-zero components of $\chi\left(T>T_{c}\right)$ in the paraelectric phase which when contracted with the order parameter give rise to all the nonzero components of $\chi^{S H G}$ in the ferroelectric phase, i.e., 
$\chi_{z z z}$ and $\chi_{x x z}(3)=\chi_{y y z}(3)((3)$ denotes all possible permutations of the 3 indices). Moreover, it follows from the above expression that the SHG susceptibility in the ferroelectric phase is a linear function of the ferroelectric order parameter.

As already mentioned earlier, $\mathrm{YMnO}_{3}$ belongs to the crystal class $6 \mathrm{~mm}$ in the ferroelectric phase. Below $T_{N}$, the six magnetic ions in the unit cell order antiferromagnetically and perpendicular to the six-fold axis, i.e. three spins are arranged in a triangular structure on planes normal to the six-fold axis and separated from each other by a distance equal to half the lattice period along the hexagonal axis. The magnetic ordering in this material is non-collinear but coplanar and can be determined from the exchange interactions among the spins only, similar to that in $\mathrm{Cr}_{2} \mathrm{O}_{3}$. The corresponding magnetic group is $\underline{6} m \underline{m}$. Following Nedlin [17] and Pashkevich et al. [18], we consider the following linear combinations of spins:

$$
\begin{aligned}
& \mathbf{s}=\mathbf{S}_{\mathbf{1}}+\mathbf{S}_{\mathbf{2}}+\mathbf{S}_{\mathbf{3}}+\mathbf{S}_{\mathbf{4}}+\mathbf{S}_{\mathbf{5}}+\mathbf{S}_{\mathbf{6}} \\
& \mathbf{l}=\mathbf{S}_{1}+\mathbf{S}_{2}+\mathbf{S}_{3}-\mathbf{S}_{4}-\mathbf{S}_{\mathbf{5}}-\mathbf{S}_{\mathbf{6}} \\
& \tau_{\mathbf{1}}=\mathbf{S}_{\mathbf{1}}-\omega^{*} \mathbf{S}_{\mathbf{2}}-\omega \mathbf{S}_{\mathbf{3}}-\mathbf{S}_{\mathbf{4}}+\omega^{*} \mathbf{S}_{\mathbf{5}}+\omega \mathbf{S}_{\mathbf{6}} \\
& \tau_{2}=-\tau_{1}{ }^{*} \\
& \sigma_{\mathbf{1}}=\mathbf{S}_{\mathbf{1}}-\omega^{*} \mathbf{S}_{\mathbf{2}}-\omega \mathbf{S}_{\mathbf{3}}+\mathbf{S}_{\mathbf{4}}-\omega^{*} \mathbf{S}_{\mathbf{5}}-\omega \mathbf{S}_{\mathbf{6}} \\
& \sigma_{2}=\sigma_{1}{ }^{*} \text {, }
\end{aligned}
$$

where $\omega^{*}$ is the complex conjugate of the phase factor $\omega=e^{i \pi / 3}$. The magnetic irreducible representations are given by some linear combinations $\psi$ of the spin components (see Table III). Since the spin ordering is coplanar, $\psi$ may easily be expressed in terms of the components of the vectors $\mathbf{s}, \mathbf{l}, \tau$ and $\sigma$ written in the cyclic coordinate frame [17,18] as follows.

$$
\mathbf{s}=e^{-} s^{+}+e^{+} s^{-}+e_{z} s^{z},
$$

where $s^{ \pm}=s^{x} \pm i s^{y}$ and similarly for $\mathbf{l}, \tau$ and $\sigma$. Here the $e_{i}$ 's are the unit vectors along the axes of the crystal coordinate frame (the z-axis coincides with the six-fold axis of the crystal lattice).

From Table [I] we learn that $\psi_{1}, \cdots, \psi_{4}$ transform according to the one-dimensional representations $A_{1}, A_{2}$, $B_{1}$ and $B_{2}$ whereas $\psi_{5}$ and $\psi_{6}$ transform according to the two-dimensional irreducible representations $E_{1}$ and $E_{2}$ of the paramagnetic and ferroelectric group $6 \mathrm{~mm}$. The irreducible representation which remains invariant under all symmetry elements of the magnetic group $\underline{6} m \underline{m}$ is $\mathrm{B}_{1}$, therefore $\psi_{3}$ is a good candidate to be defined as the antiferromagnetic order parameter [18] for $\mathrm{YMnO}_{3}$.

$\psi_{3}$ is a complicated combination of the spin components of the six ions in the unit cell. Let us build a more intuitive object which belongs to the same $B_{1}$ irreducible representation and where not only spin components but also vector components are introduced, whose physical interpretation may be that of a local field on each $\mathrm{Mn}^{3+}$ ion. Thus, similar to the spins $\mathbf{S}_{\mathbf{1}} \cdots \mathbf{S}_{\mathbf{6}}$, one can introduce i-polar vectors $\mathbf{V}_{\mathbf{1}} \cdots \mathbf{V}_{\mathbf{6}}$ (which might correspond to local planar displacements of the $\mathrm{Mn}^{3+}$ ions) and form the linear combinations $\mathbf{p}, \mathbf{q}, \eta$ and $\rho$ analogous to $\mathbf{s}, \mathbf{l}$, $\tau$ and $\sigma$ in Eq. (13). These linear combinations of the i-polar vectors also follow the irreducible representations of the spatial group $6 \mathrm{~mm}$. From the direct product representations of the spin pseudovectors and that of the i-polar vectors (which of course is reducible), one obtains the following combination $\Lambda=\sigma_{1}^{+} \eta_{2}^{-}+\sigma_{2}^{-} \eta_{1}^{+}$which becomes invariant under all the symmetry operations of the magnetic group. Thus $\Lambda$ should be equivalent to $\psi_{3}$ in the sense that they belong to the same irreducible one dimensional representation. Furthermore, from the generating matrices of the magnetic group, one can also construct an invariant c-axial quantity in lowest order, which in the present case is a tensor of rank three, $R_{3}$ (in $\underline{6} m \underline{m}$, all the c-axial tensors of rank smaller than three vanish). Now expanding the free energy in terms of $R_{3}$ one obtains in lowest order,

$$
\begin{gathered}
F=-\left[\chi_{i j k l m n}\left(T>T_{N}\right) E_{i}^{*} E_{j} E_{k}\right. \\
\left.+\chi_{i j k l m n}^{*}\left(T>T_{N}\right) E_{i} E_{j}^{*} E_{k}^{*}\right] R_{l m n}
\end{gathered}
$$

and thus the SHG susceptibility tensor can be obtained as,

$$
\chi_{i j k}^{S H G}\left(T<T_{N}\right)=\chi_{i j k l m n}\left(T>T_{N}\right) R_{l m n}
$$

It should be noted that the c-axial tensor $R_{3}$ has only one independent component, i.,e., $R_{y y y}=-R_{x x y}(3)$ which can be identified as the order parameter discussed above. Performing the sum over $l, m$ and $n$ in Eq. (16) explicitly we then obtain an alternative expression for the allowed matrix elements contributing to SHG in the antiferromagnetic phase as

$$
\begin{gathered}
\chi_{i j k}^{S H G}\left(T<T_{N}\right)= \\
{\left[\chi_{i j k y y y}\left(T>T_{N}\right)-\chi_{i j k x x y}\left(T>T_{N}\right)\right.} \\
\left.-\chi_{i j k x y x}\left(T>T_{N}\right)-\chi_{i j k y x x}\left(T>T_{N}\right)\right] \psi_{3}
\end{gathered}
$$

Thus, from the symmetry properties of the sixth rank i-axial tensor $\chi_{i j k l m n}$ above $T_{N}$ [19], together with Eq. (17), we get all the non-zero components of the SHG susceptibility tensor for $T<T_{N}$, which are, $\chi_{x x x}^{S H G}=$ $-\chi_{y y x}^{S H G}=-\chi_{y x y}^{S H G}=-\chi_{x y y}^{S H G}$ (as one can easily verify, see [19]).

Since $\mathrm{YMnO}_{3}$ is characterized by two order parameters, one for the paraelectric-ferroelectric transition and the other for the paramagnetic-antiferromagnetic transition, it is natural to ask about the coupling between them. We observe here that the SHG tensor in the antiferromagnetic phase, as given by Eq. (17), is directly proportional to the even-rank i-axial tensor $\chi_{i j k l m n}$ which is not allowed in the high-temperature group $6 / \mathrm{mmm}$ for $T>T_{c}$, which is centrosymmetric. $\chi_{i j k l m n}$ need therefore to be proportional to the ferroelectric order parameter (compare Eq. (11) and Eq. (12)):

$$
\chi_{i j k l m n}\left(T>T_{N}\right)=\chi_{i j k l m n z}\left(T>T_{c}\right) P_{z}^{(S P)},
$$


where $\chi_{i j k l m n z}$ is an i-axial tensor of rank seven which is allowed in $6 / \mathrm{mmm}$. Comparing Eq. (18) with Eq. (17) we find immediately:

$$
\begin{gathered}
\chi_{i j k}^{S H G}\left(T<T_{N}\right)=\chi_{i j k l m n z}\left(T>T_{c}\right) R_{l, m, n} P_{z}^{(S P)}= \\
{\left[\chi_{i j k y y y z}\left(T>T_{c}\right)-\chi_{i j k x x y z}\left(T>T_{c}\right)\right.} \\
\left.-\chi_{i j k x y x z}\left(T>T_{c}\right)-\chi_{i j k y x x z}\left(T>T_{c}\right)\right] \psi_{3} P_{z}^{(S P)} .
\end{gathered}
$$

It is therefore possible to derive all the components of the SHG susceptibility tensor below $T_{N}$ from the non-zero components of $\chi_{i j k l m n o}$ together with both the order parameters. It is also clear from the above equation that the SHG susceptibility below $T_{N}$ is directly proportional to the bilinear combination of both order parameters which in principle, could be verified from experiments.

The method described in the present manuscript to study the nonreciprocal optical effects in the magnetic materials through SHG is purely phenomenological and based on symmetry considerations. Therefore, it should be possible to generalize this phenomenology to other hexagonal rare-earth manganites $\mathrm{RMnO}_{3}$ where $\mathrm{R}=\mathrm{Ho}$, Er, Tm, Yb, Lu etc.

In conclusion, we summarize the main findings of the present paper. A phenomenological Ginzburg-Landau theory has been developed for second harmonic generation in materials which undergo one or more phase transitions by expanding the free energy in terms of the order parameter/s and the susceptibility tensor in the high-temperature phase. We have shown how to obtain explicitly the SHG susceptibility components as a function of certain susceptibility tensors allowed in the high-temperature phase and of the order-parameter. We have carried through this prescription for the magnetoelectric compound $\mathrm{Cr}_{2} \mathrm{O}_{3}$ as well as for the ferroelectricantiferromagnetic material $\mathrm{YMnO}_{3}$. We also argue that this analysis can be extended to the other hexagonal rareearth manganites.

\section{ACKNOWLEDGMENTS}

The authors would like to acknowledge several discussions with D. Fröhlich, R. V. Pisarev, St. Leute, Th. Lottermoser, C. Reimpell and M. Fiebig. This work is supported by the Deutsche Forschungsgemeinschaft, the Graduiertenkolleg "Festkörperspektroskopie".

† E-mail: debanand@cip2.physik.uni-dortmund.de

[1] Y. R. Shen, "The Principles of Non-linear Optics", Wiley, New York (1984).

[2] B. B. Krichevtsov, V. V. Pavlov, R. V. Pisarev and V. N. Gridnev, J. Phys. Cond. Matter 5, 8233, (1993).

[3] M. Fiebig, D. Fröhlich, B. B. Krichevtsov, and R. V. Pisarev, Phys. Rev. Lett. 73, 2127, (1994).

[4] M. Fiebig, D. Fröhlich and G. Sluyterman v. L., Appl. Phys. Lett. 66, 2906, (1995).

[5] V. N. Muthukumar, R. Valenti and C. Gros, Phys. Rev. Lett. 75, 2766, (1995), Phys Rev. B 54, 433, (1996).

[6] Y. Tanabe, M. Muto and E. Hanamura, Solid State Comm. 102, 643 (1997); M. Muto, Y. Tanabe, T. IizukaSakano and E. Hanamura, Phys. Rev. B 57, 9586 (1998).

[7] V. L. Ginzburg and L. D. Landau, Zh. Eksp. Teor. Fiz. 20, 1064 (1950).

[8] P. S. Pershan, Phys. Rev. 130, 919, (1963).

[9] J. C. Tolédano and P. Tolédano, "The Landau Theory of Phase Transitions", World Scientific, Singapore (1987).

[10] R. R. Birss, "Symmetry and Magnetism", North-Holland, Amsterdam (1966).

[11] I. E. Dzialoshinskii, Sov. Phys. JETP, 5, 1259, (1957).

[12] M. Tinkham, "Group Theory and Quantum Mechanics", McGraw-Hill (1964).

[13] Landolt-Börnstein, "Numerical data and Functional Relationships, New Series, III/16a", Springer-Verlag, Berlin (1981).

[14] Z. J. Huang, Y. Cao, Y. Y. Sun, Y. Y. Xue and C. W. Chu, Phys. Rev. B 56, 2623, (1997).

[15] M. N. Iliev, N. -G. Lee, V. N. Popov, M. V. Abrashev, A. Hamed, R. L. Meng and C. W. Chu, Phys. Rev. B 56, 2488, (1997).

[16] D. Fröhlich, St. Leute, V. V. Pavlov and R. V. Pisarev, Phys. Rev. Lett. 81, 3239, (1998).

[17] G. M. Nedlin, Sov. Phys. Sol. St. 6, 2156, (1965).

[18] Yu. G. Pashkevich, V. L. Sobolev, S. A. Fedorov and A. V. Eremenko, Phys. Rev. B 51, 15898, (1995).

[19] R. Fieschi and F. G. Fumi, Nouvo Cimento 10, 865, (1953). 


\begin{tabular}{c|c|} 
& Irreducible Representations \\
\hline$A_{2 g}$ & $m_{z}$ \\
\hline$A_{1 g}$ & $l_{1 z}$ \\
\hline$A_{1 u}$ & $l_{2 z}$ \\
\hline$A_{2 u}$ & $l_{3 z}$ \\
\hline$E_{g}$ & $\left(\begin{array}{c}m_{x}, m_{y} \\
l_{1 x}, l_{1 y}\end{array}\right)$ \\
\hline$E_{u}$ & $\left(\begin{array}{l}l_{2 x}, l_{2 y} \\
l_{3 x}, l_{3 y}\end{array}\right)$ \\
\hline
\end{tabular}

TABLE I. Classification of the components of the vectors $m, l$, in accordance with the irreducible representations of the crystallographic point group $\overline{3} m$.

\begin{tabular}{c|c|} 
& Irreducible Representations \\
\hline$A_{1}$ & $\psi_{1}=-\left(\tau_{1}^{-}+\tau_{2}^{+}\right)$ \\
\hline$A_{2}$ & $\psi_{2}=\left(\tau_{1}^{-}-\tau_{2}^{+}\right)$ \\
$\psi_{2}^{\prime}=s^{z}$ \\
\hline$B_{1}$ & $\psi_{3}=\left(\sigma_{1}^{-}+\sigma_{2}^{+}\right)$ \\
\hline$B_{2}$ & $\psi_{3}^{\prime}=l^{z}$ \\
\hline$E_{1}$ & $\left(\psi_{4}=\left(-\sigma_{1}^{-}+\sigma_{2}^{+}\right)\right.$ \\
\hline & $\left(\psi_{5, I I I}=s^{+}, \psi_{5, I I}=s_{2}^{-}, \psi_{5, I V}=\sigma_{1}^{+}\right)$ \\
\hline$E_{2}$ & $\left(\psi_{5, V}=\tau_{1}^{z}, \psi_{5, V I}=-\tau_{2}^{z}\right)$ \\
\hline & $\left(\psi_{6, I}=l^{+}, \psi_{6, I I}=-\tau_{2}^{-}, \psi_{6, I V}=\tau_{1}^{+}\right)$ \\
\hline
\end{tabular}

TABLE II. Classification of the components of the vectors $s, l, \tau$ and $\sigma$ in accordance with the irreducible representations of the crystallographic point group $6 \mathrm{~mm}$. 